\title{
CONTRIBUTO ALLA TEORIA DEI PRODOTTI INFINITI E DELLE SERIE A TERMINI POSITIVI.
}

Nota di Ettore Bortolotti, in Modena.

Adunarza del is marzo 1904 .

Mi propongo, con questo breve lavoro, di cercare delle relazioni fra il comportamento assintotico della variabile $\varphi_{n}=\prod_{r=1}^{n}\left(\mathrm{I}+a_{r}\right) \mathrm{e}$ la legge di formazione della variabile reale $a_{n}$, quando $n$ tenda allinfinito.

Porrò prima in riscontro le variabili $\varphi_{n}$, ed $e^{\sum_{\Sigma}^{n} a_{r}}$, ricavandone, per la $\varphi_{n}$, le condizioni di convergenza assoluta e condizionata, e, nel caso della convergenza, un metodo per il calcolo approssimato, nel caso della divergenza, un criterio per determinarne la rapidità di crescenza.

In secondo luogo, ponendo $a_{n}^{\prime}+a_{n}^{\prime \prime}=a_{n}$, cercherò di far dipendere la determinazione del comportamento assintotico, od il calcolo numerico nel caso della convergenza, della variabile $\varphi_{n}=\prod_{1}^{n}\left(1+a_{r}\right)$, da quello delle variabili $\psi_{n}^{\prime}=\prod_{\mathrm{I}}^{n}\left(\mathrm{I}+a_{r}^{\prime}\right), \psi_{n}^{\prime \prime}=\prod_{\mathrm{I}}^{n}\left(\mathrm{I}+a_{r}^{\prime \prime}\right)$.

Sard agevole di qui passare al caso in cui si abbia $a_{n}=\alpha b_{n}$, e stabilire analoghe relazioni fra le variabili $\varphi_{n}=\prod_{1}^{n}\left(\mathrm{I}+a_{r}\right), \psi_{n}=\prod_{1}^{n}\left(\mathrm{I}+b_{r}\right)$.

In particolare si vedrà che, nel caso della divergenza, se l'ordine di infinito (di infinitesimo) della variabile $\psi_{n}$ si assume come unità, e se $\alpha$ è una costante positiva, l'ordine della $\varphi_{n}$ è espresso dal simbolo $(\alpha)$.

Più generalmente, se $a_{n}, c_{n}$ sono variabili reali, soddisfacenti le con- 
dizioni: $\alpha$ ) le $a_{n}$ sono tutte dello stesso segno, $(\beta) \imath$ binomi $\mathrm{I}+a_{n} \mathrm{I}+a_{n} c_{n}$ sono tutti posativa, $e$ se $\beta_{1}, \beta_{2}$ sono $i$ limiti inferiore e superiore di indeter minazione della espressione $\frac{\lg \left(1+a_{n} c_{n}\right)}{\lg \left(1+a_{n}\right)}$, la variabule $\prod_{\mathrm{I}}^{n}\left(\mathrm{I}+a_{n} c_{n}\right)$ non è infinita (unfintestma) di ordnne inferwore a quello della varabile $\left[\prod_{1}^{n}\left[\left(\mathrm{I}+a_{r}\right)\right]^{\beta_{1}-\varepsilon}\right.$, ne di ordine siperiore a quello della variabile $\left[I_{\mathbf{I}}^{n}\left(\mathrm{I}+a_{r}\right)\right]^{\beta_{\mathbf{I}}+\varepsilon}$, $\varepsilon$ positivo qualunque.

In particolare, se $\lim _{n=\infty} a_{n}=0, \operatorname{limi}_{n=\infty} c_{n}=\beta$, st ba $\beta_{\mathrm{I}}=\beta_{2}=\beta$, e cioé, l'ordine dimfinito della $\prod_{1}^{n}\left(\mathrm{I}+a_{r} c_{r}\right)$, secondo il concetto di CaUCHY, è eguale a quello della variabile $\left[\prod_{1}^{n}\left(\mathrm{I}+a_{r}\right)\right]^{\beta}$.

Riprendendo alcune ricerche precedenti, $m i$ studierò in fine di mettere in relazione le variabili

$$
\varphi_{n}=\prod_{r=x}^{n}\left[1+a\left(x_{0}+n\right)\right], \quad f(n)=e^{\int_{\lambda_{0}}^{\alpha_{0}+n} a(x) d x},
$$

estendendo cosi ai prodotti infiniti un metodo che fu dato dal Cauchy per le serie a termini positivi.

D1 cotesta quistione, per altro, non darò che un rapido cenno, rıserbando ad un prossimo studio una plù ampia trattazione

Ho voluto nel $\S I$, insieme con alcuni risultamenti che ritengo nuovi, stabilire le principali proposizıoni che servono di fondamento alla teoria de1 prodott infinitı, perchè il metodo che ho seguito porta a dimostrarle, quasi senza sviluppi di calcolo, nel modo che parmi più semplice e naturale ${ }^{*}$ ).

*) Non richiedo a chi legge che le prime nozion sui limiti e sulle serie, qualı si insegnano nel corso di Algebra ai nostrı studentl di primo anno e s1 trovano, per fissare le idee, nell'Analtsi Algebrica del Cesàro.

Il Pringsheim in una dotta memoria del vol. XXXIII dei "Mathematische Annalen" stabilisce 1 fondamentr sulla convergenza dei prodotti infiniti con metodo certo rigoroso, e, dal suo punto di vista, elementare; ma lungo, difficle, laboriosissimo. Egh trova necessario quel metodo, per evitare la considerazıoue di trascendentı logaritmiche, usate dal Dris nella sua classica memona "Su prodoth unfuntı" (Annali di Matematica, sene II, t. 2, I87o). Credo che il $\$$ I della presente pubblicazıone, dove mi sono studiato 
Delle serie a termin posttu, tratto incidentalmente, nel $\$$ II, per mostrare come il metodo che uso per lo studio dei prodota infiniti, possa giovare anche per quello delle serie, e conduca rapidamente ai criteri, detii logaritmici, di convergenza, ed a criterî che in taluni casi servono per determinare l'o dine di infinito di serie divergenti. Nel $\$$ III, e nel IV, poi, studio il comportamento assintonco della serie a termini positivi $\sum\left[a_{n}-\lg \left(\mathrm{I}+a_{n}\right)\right]$, dove $a_{n}$ è una variabile reale infinitesima per $n=\infty$.

Questa serie, per $a_{n}=\frac{I}{n}$, ha per somma la costante di Eulero ed è in ogni caso strettameite legata con le variabili

$$
\varphi_{n}=\prod_{r=1}^{n}\left(\mathrm{I}+a_{r}\right), \quad A_{n}=\sum_{r=1}^{n} a_{r} .
$$

\section{SI.}

I. Sia $a_{r}$ una variabule positiva; si indichi con $A_{n}$ la somma dei primi termini della serie

$$
\sum_{r}^{\infty} a_{r}=a_{r}+a_{2}+\cdots
$$

e con $\varphi_{n} 11$ prodotto dei primi $n$ fattori nel prodotto infinito:

$$
\mathbb{1}_{\mathrm{r}}^{\infty}\left(\mathrm{I}+a_{r}\right)=\left(\mathrm{I}+a_{\mathrm{I}}\right)\left(\mathrm{I}+a_{2}\right) \ldots
$$

Le variabili $A_{n}, \varphi_{n}$ sono entrambe sempre crescentr; se una alneno di esse è infinita per $u=\infty$, potren. scrivere ${ }^{*}$ ):

$$
\lim _{n=\infty} \frac{A_{n}}{\lg \varphi_{n}}=\lim _{n=\infty} \frac{A_{n}-A_{n-1}}{\lg \psi_{n}-\lg \varphi_{n-1}},
$$

quando esista il secondo membro.

Ma si ha

$$
\lim _{n=\infty} \frac{A_{n}-A_{n-1}}{\lg \varphi_{n}-\lg \varphi_{n-1}}=\lim _{n=\infty} \frac{1}{\lg \left(1+a_{n}\right)^{\frac{1}{a_{n}}}}
$$

di imitare la scioltezza e la eleganza der metodi del Dini e del Cauchy, evitando considerazion di ordine trascendcatule, non sara trovato dai nostri scolarn meno elementare di quello del Pringsheim.

*) Cfr. Cesàro, Analisi Algebrica, pag 98. 
dunque, quando sia determinato il limite della espressione $\lg \left(\mathrm{I}+a_{n}\right)^{\frac{1}{a_{n}}}$, avremo :

$$
\lim _{n=\infty} \frac{A_{n}}{\lg \varphi_{n}}=\lim _{n=\infty} \frac{\mathrm{I}}{\lg \left(\mathrm{I}+a_{n}\right)^{\frac{\mathrm{I}}{a_{n}}}} ;
$$

ed, in ogni caso, usando il simbolo $\lim _{n=\infty} \operatorname{nnf} f_{n}$ per indicare il limite niferore di indeterminazione della varibile $f_{n}$, si avrà ${ }^{*}$ ):

$$
\liminf _{n=\infty} \frac{A_{n}}{\lg \varphi_{n}} \supseteq \liminf _{n=c} \frac{\mathrm{I}}{\lg \left(\mathrm{I}+a_{n}\right)^{\frac{1}{a_{n}}}} .
$$

2. Supponiamo ora che sia

$$
\lim _{n \rightarrow \infty} a_{n}=0 \text {. }
$$

Per ogni valore finito di $u$ avremo $\left(1+a_{n}\right)^{\frac{1}{a_{n}}}<e$, ed al limite, per $\left.n=\infty, \lim _{n=\infty}\left(\mathrm{I}+a_{n}\right)^{\frac{1}{a_{n}}}=e^{* *}\right)$.

D'onde si deduce:

ed anche:

$$
\frac{A_{n}}{\lg \varphi_{n}}=\mathrm{I}+\varepsilon_{n}, \quad \varepsilon_{n}>0, \quad \lim _{n=\infty} \varepsilon_{n}=0
$$

$$
\frac{e^{A_{n}}}{\varphi_{n}}=\varphi_{n}^{\varepsilon_{n}}, \quad \varepsilon_{n}>0, \quad \lim _{n=\infty} \varepsilon_{n}=0 .
$$

3. Le variabili sempre crescenti $A_{n}, \varphi_{n}$ sono determinate per $n=\infty$.

Se non è $\lim _{n=\infty} a_{n}=0$, divergono entrambe, se è $\lim _{n=\infty} a_{n}=0$ possono convergere. La formula (8) però, dimostra che esse sono sempre insieme divergenti: d'onde il noto teorema:

Condizione necessaria e sufficiente per la convergenza del prodotto infinito $\prod_{\mathrm{r}}^{\infty}\left(\mathrm{I}+a_{n}\right) \dot{e}$ che converga la serie $\sum_{\mathbf{1}}^{\infty} a_{n}$.

4. Le variabili $A_{n}, \varphi_{n}$ sieno convergent. Posto

$$
\text { (9) } \alpha_{n}=a_{n+1}+a_{n+2}+\cdots \quad Q_{n}=\left(\mathrm{I}+a_{n+1}\right)\left(\mathrm{I}+a_{n+2}\right) \ldots \text {, }
$$

*) Cfr. O. Stolz, Mathematische Annalen, Bd. XXXIII, pag. 243.

**) Cesàro, loc. cit., pag. Iro. 
avremo *)

(IO) $\lim _{n=\infty} \frac{\alpha_{12}}{\lg Q_{n}}=\lim _{n=\infty} \frac{\alpha_{n}-\alpha_{n+1}}{\lg Q_{n}-\lg Q_{n+1}}=\lim _{n=\infty} \frac{a_{n+1}}{\lg \left(\mathrm{I}+a_{n+1}\right)}=\mathrm{I}$.

Scriveremo perciò

(II) $\alpha_{n}=\lg Q_{n}^{1+\varepsilon_{n}}, \quad \frac{e^{\alpha_{n}}}{Q_{n}}=Q_{n}^{\varepsilon_{n}}, \quad \varepsilon_{n}>0, \quad \lim _{n=\infty} \varepsilon_{n}=0$

ed anche

$$
e^{\sum_{r=n}^{\infty} a_{r}}>\prod_{r=n}^{\infty}\left(\mathrm{I}+a_{r}\right), \quad \lim _{n=\infty} \frac{e^{\sum_{r=n}^{\infty} a_{r}}}{\prod_{r=n}^{\infty}\left(\mathrm{I}+a_{r}\right)}=\mathbf{I} .
$$

La variablle $\Gamma_{\mathrm{r}}\left(\mathrm{I}+a_{r}\right)$ ba dunque la stessa rapidità di tendenza al limite della variabile $e^{\sum_{\mathrm{r}}^{n} a_{r}}$.

Calcolando direttamente il prodotto dei primi $n$ fattori, avremo un limite superiore dell'errore commesso trascurando gli altri, se calcoleremo la espressione $e^{\sum_{n}^{\infty} a_{\gamma}}$, od una espressione approssimata in eccesso di questa.

5. Nel caso della divergenza, le formule (7) ci assicurano che la serie $\sum_{i}^{\infty} a_{r}$ ba lo stesso ordine de infinto e la stessa parte principale del logaritmo del prodotto infinato $\prod_{1}^{\infty}\left(1+a_{r}\right)$.

Dalle formule (8) poi ricaviamo

$$
\lim _{n=\infty} \frac{e^{A_{n}}}{\varphi_{n}} \supseteq \mathrm{I}
$$

cioè l'ordine di infinito del prodotto $\prod_{1}^{\infty}\left(\mathbf{I}+a_{r}\right)$ è minore od eguale a quello della espressione $e^{\sum_{\Sigma}^{\infty} a_{r}}$.

Al $₫$ III vedremo in quali casi cotesti ordini sieno eguali.

*) Ibidem, pag. 99. 
6. Indicando con $b_{n}$ una variabile positiva infinitesima per $n=\infty$, e con $B_{n}$ la somma $B_{n}=b_{1}+b_{2}+\ldots+b_{n}$, poniamo:

$$
\psi_{n}=\left(\mathrm{I}-b_{\mathrm{s}}\right)\left(\mathrm{I}-b_{2}\right) \ldots\left(\mathrm{I}-b_{n}\right) \text {. }
$$

Le variabili $B_{n}, \psi_{n}$, sono entrambe monotone; nel caso che la prima sia infinita, o che la seconda sia infinitesima, potremo scrivere:

$$
\lim _{n=\infty} \frac{B_{n}}{\lg \psi_{n}}=\lim _{n=\infty} \frac{B_{n}-B_{n-1}}{\lg \psi_{n}-\lg \psi_{n-1}}=\lim _{n=\infty} \frac{\mathbf{I}}{\lg \left(\mathrm{I}-b_{n}\right)^{\frac{1}{b_{n}}}}=\text { - I . }
$$

Se $\operatorname{con} \sigma_{n}$ indichiamo una variabile positiva infinitesima per $n=\infty$, avremo perciò

$$
\text { (I4) } \frac{-B_{n}}{\lg \psi_{n}}=\mathrm{I}-\sigma_{n}, \frac{e^{-B_{n}}}{\psi_{n}}=\psi_{n}^{-\sigma_{n}}, \quad \sigma_{n}>0, \lim _{n=\infty} \sigma_{n}=0 \text {. }
$$

Ricordando che per le variabili monotone $B_{n}, \psi_{n}$, esiste sempre limite determinato, ricaveremo di qui la proposizione:

Condizione necessaria e sufjiciente perchè il prodotto infinto $\left(\mathrm{I}-b_{n}\right)$ converga verso un limite diverso dallo zero, è la convergenza della serie $\sum_{i}^{\infty} b_{n}$.

Inoltre potremo affermare che:

Se la serie $\sum_{1}^{\infty} b_{12}$ diverge, il prodotto infinito $\prod_{1}^{\infty}\left(1-b_{n}\right)$ è infinitesimo di ordine maggiore od eguale a quello della espressione $e^{-\sum_{1}^{\infty} b_{n}}$.

7. Sia $c_{n}$ una variabile reale infinitesima per $n=\infty$.

Consideriamo il prodotto infinito $\prod_{I}^{\infty}\left(I+c_{n}\right)$.

Se nella serie $\sum_{I}^{\infty} c_{n}$ distinguiamo coi simboli $a_{1}, a_{2}, a_{3}, \ldots a_{m}, \ldots$ $b_{1}, b_{2}, b_{3}, \ldots b_{m}, \ldots$ i termini positivi ed $\mathrm{i}$ valori assoluti dei termini negativi rispettivamente, potremo distinguere $\mathrm{i}$ tre seguenti casi ${ }^{*}$ ):

I. Le serie $\sum a_{m}, \sum b_{m}$, convergono entrambe.

II. $" ~ » ~ »$ divergono entrambe.

III. " » $\gg$ sono l'una convergente, l'altra divergente.

*) Cfr. DiNi, Suz prodott infinti, Annali di Matematica, serie II, tomo II, pag. 3 I. 
1). Se le serie $\sum a_{n}, \sum b_{n}$ convergono entrambe, sono convergenti anche $i$ prodotti infiniti $\prod\left(\mathrm{I}+a_{n}\right), \prod\left(\mathrm{I}-b_{n}\right)$ e la serie $\sum c_{n}$ converge assolutamente.

Se indichiamo con $c_{1}^{\prime}, c_{2}^{\prime}, c_{3}^{\prime}, \ldots$ una particolare permutazione dei termini della $\sum c_{n}$, ad ogni coppia di numeri positivi $\varepsilon, r$ faremo corrispondere un numero $n_{0}$ tale che le somme

$$
a_{n_{\mathrm{S}}}^{\prime}+a_{n_{\mathrm{S}}+\mathrm{r}}^{\prime}+\cdots+a_{n_{\mathrm{1}}+r_{1}}^{\prime}, b_{n_{2}}^{\prime}+b_{n_{2}+\mathrm{I}}^{\prime}+\cdots+b_{n_{2}+r_{2}}^{\prime}
$$

dei termini positivi e dei valori assoluti dei termini negativi contenuti nella somma :

$$
c_{n}^{\prime}+c_{n+1}^{\prime}+\cdots+c_{n+r}^{\prime}, \quad n \supseteq n_{0},
$$

sieno entrambe minori di $\varepsilon$.

Ma si ha:

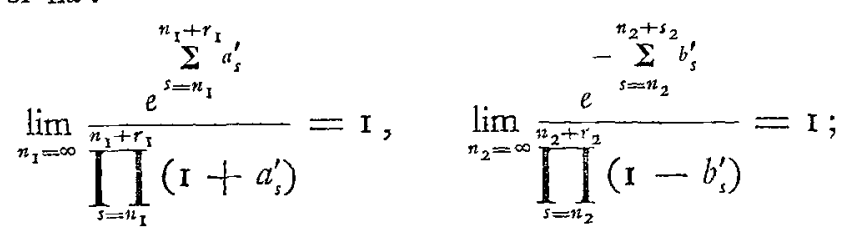

faremo dunque corrispondere al numero $\varepsilon$ un numero $N$ maggiore di $n_{\mathrm{o}}$ e tale che

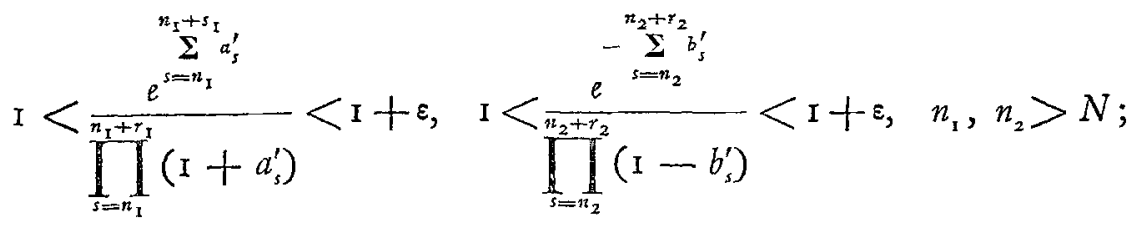

cioè :

$$
\left.\frac{\mathrm{I}}{\mathrm{I}+\varepsilon}<\prod_{s=n_{1}}^{n_{1}+r_{I}}\left(\mathrm{I}+a_{s}^{\prime}\right)<e^{\varepsilon}, \quad \frac{e^{-\varepsilon}}{\mathrm{I}+\varepsilon}<\prod_{\frac{s=n_{2}}{n_{2}+r_{2}}} \prod_{1}-b_{s}^{\prime}\right)<\mathrm{I} ;
$$

finalmente :

$$
\frac{e^{-\varepsilon}}{(\mathrm{I}+\varepsilon)^{2}}<\prod_{s=n}^{n+r}\left(\mathrm{I}+c_{s}^{\prime}\right)<e^{\varepsilon}, \quad n>N
$$

e ciò dimostra la convergenza del prodotto $\left.\prod_{\mathbf{I}}^{\infty}\left(\mathrm{I}+c_{n}^{\prime}\right)^{*}\right)$, cioè la convergenza assoluta del prodotto $\prod_{\mathrm{I}}^{\infty}\left(\mathrm{I}+c_{n}\right)$.

*) Cfr. p. es. Pringsheim, Mathematische Annalen, XXXIII, pp. I27, i 28. 
II. Le serie $\sum a_{n}, \sum b_{n}$ divergono entrambe. Cangiando l'ordine dei termini nelle serie $\sum c_{n}$ in tutti i modi possibili, questa serie verrà a prendere tutti i valor da $+\infty$ a $-\infty$, e clascuno un numero infinito di volte; e per dati cangiamenti dello stesso ordine diverrà anche indeterminata.

Ordiniamo $i$ termini $c_{n}$ in modo che la somma $c_{1}^{\prime}+c_{2}^{\prime}+\cdots+c_{n}^{\prime}$, per $n$ abbastanza grande, superi qualunque numero positivo $M$.

Conservando le notazioni usate nel caso precedente, avremo

$$
\sum_{s=1}^{n_{1}} a_{s}^{\prime}-\sum_{s=1}^{n_{2}} b_{s}^{\prime}>M, \quad n \geqq n_{0}
$$

Dunque

$$
n \geqslant n_{0}, \quad \frac{\sum_{s=1}^{n_{1}} a_{s}^{\prime}}{\sum_{s=1}^{n_{2}} b_{s}^{\prime}}>\mathrm{I}+\frac{M}{\sum_{s=1}^{n_{2}} b_{s}^{\prime}}
$$

e potremo supporre le $c_{n}^{\prime}$ ordinate in modo che 1 quoziente $\frac{M}{\sum_{1}^{n_{2}} b_{s}^{\prime}}$ abbia limite superiore di indeterminazione maggiore di zero.

$\mathrm{Da}$ ciò, e dalle relazioni

$$
\lim _{n_{\mathrm{I}}=\infty} \frac{\sum_{s=1}^{n_{\mathrm{T}}} a_{s}^{\prime}}{\lg \prod_{\mathrm{I}}^{n_{\mathrm{I}}}\left(\mathrm{I}+a_{s}^{\prime}\right)}=\mathrm{I}, \quad \lim _{n_{2}=\infty} \frac{-\sum_{\mathrm{I}}^{n_{2}} b_{s}^{\prime}}{\lg \prod_{\mathrm{I}}^{n_{2}}\left(\mathrm{I}-b_{s}^{\prime}\right)}=\mathrm{I}
$$

[formule (7), (r 4$)$, dedurremo immediatamente :

$$
\lim _{n=\infty} \frac{\sum_{s}^{n_{1}} a_{s}^{\prime}-\sum_{I}^{n_{2}} b_{s}^{\prime}}{\lg \prod_{1}^{n_{1}}\left(1+a_{s}^{\prime}\right)+\lg \prod_{1}^{n_{2}}\left[\left(1-b_{s}^{\prime}\right)\right.}=\mathrm{I},
$$

cioè

$$
\lim _{n=\infty} \frac{\sum_{s=1}^{n} c_{s}^{\prime}}{\lg \prod_{s}^{n}\left(\mathrm{I}+c_{s}^{\prime}\right)}=\mathrm{I}
$$

e ciù prova che, nell'ordine indicato, il prodotto $\prod_{\mathrm{I}}^{\infty}\left(\mathrm{I}+c_{\mathrm{s}}^{\prime}\right)$ diverge verso $+\infty$. 
La medesima dimostrazione, quando la serie $\sum c_{12}$ sia ordinata in modo che la somma $c_{1}^{\prime \prime}+c_{2}^{\prime \prime}+\cdots+c_{12}^{\prime \prime}$ si mantenga inferiore di qualunque numero negativo $-M$, serve a provare che il prodotto infinito $\frac{1}{1}\left(\mathrm{I}+c_{n}^{\prime \prime}\right)$ corrispondente tende al limite zero.

III. Le serie $\sum a_{n}, \sum b_{n}$, sieno l'una convergente, l'altra divergente.

La serie $\sum c_{n}$ in tal caso diverge assolutamente : con ragionamento poco diverso dal precedente, proveremo che il prodotto $\prod_{\mathrm{I}}^{\infty}\left(\mathbf{I}+c_{n}\right)$ diverge assolutamente; e cioè: verso $+\infty$ se la serie $\sum a_{n}$ diverge, verso lo zero se diverge la serie $\sum b_{n}{ }^{*}$ ).

8. Da tutto quanto abbiamo detto si ricava: Condızione necessaria e sufficiente per la convergenza assoluta del prodotto infinito $\sum_{\mathbb{L}^{\prime}}^{\infty}\left(1+c_{n}\right)$ è la convergenza assoluta della serie $\left.\sum_{\mathrm{r}}^{\infty} c_{n}^{* *}\right)$.

La condizione necessaria e sufficiente per la convergenza assoluta di una serie $\sum c_{n}$ consiste però, come è noto, nella convergenza della serie dei valori assoluti: $\sum\left|c_{n}\right|$, ed è questa anche condizione necessaria e sufficiente per la convergenza del prodotto infinito $\prod_{\mathrm{I}}^{\infty}\left[\mathrm{I}+\left|c_{n}\right|\right]$; concludiamo dunque :

Condizione necessaria e suffictente per la convergenza assoluta del prodotto infinto $\prod_{\mathrm{I}}^{\infty}\left(1+c_{n}\right)$, è la convergenza del prodotto:

*) Non mi dilungo in moltı particoları trattandosi dı cose note Il Dins, nella memoria citata, dumostra di più che alteiando l'ordine des fatiori si puó far prendere al prodotto infinito qualunque valore e anche ridurlo indeterminato.

Nel $\$ III, studierò la convergenza condızınata, considerando così il problema da un punto di vista più generale.

**) E quasi superfluo avvertire che la convergenza de un prodotto infinito si intende al modo indicato dal Dini ed esplicitamente definito dal Pringsheim, cioè si fa dipendere dalla esistenza del limite finito e diverso dallo zero per la successione

$$
P_{n}=\left(\mathrm{I}+c_{\mathrm{r}}\right)\left(\mathrm{I}+c_{2}\right) \ldots\left(\mathrm{I}+c_{n}\right), n=\mathrm{I}, 2,3, \ldots
$$




$$
\prod_{i}^{\infty}\left(\mathrm{I}+k_{n} \mid\right)
$$

formato coi valor assoluti $\left|c_{n}\right|$ delle variabili $c_{n}$.

\section{$\S$ II.}

9. Le presenti ricerche hanno principalmente in vista la teoria dei prodotti infiniti; ma i teorem or ora dimostrat ed il metodo seguito possono utilmente servire anche allo studio delle serre a termini positivi.

Servano d'esempio le proposizioni seguent1:

I. Se la varabule $\varphi_{n}$ tende allinfintu sempre crescendo e soddisfo la condizione

le due serie:

$$
\lim _{n=\infty} \frac{\varphi_{n+1}}{\varphi_{n}}=\mathrm{I}
$$

$$
\sum_{\mathrm{T}}^{\infty} a_{n}=\sum_{\mathrm{T}}^{\infty} \frac{\varphi_{n+\mathrm{I}}-\varphi_{n}}{\varphi_{n}}, \quad \sum_{\mathrm{T}}^{\infty} b_{n}=\sum_{\mathrm{T}}^{\infty} \frac{\varphi_{n+\mathrm{r}}-\varphi_{n}}{\varphi_{n+\mathrm{I}}},
$$

divergono entrambe con rapidità par a quella della varabile $\lg \varphi_{n}$.

E più esattamente si hanno le relazioni:

$$
\lim _{n=\infty} \frac{\sum_{\mathrm{I}}^{n} a_{n}}{\lg \varphi_{n}}=\mathrm{I}, \quad \lim _{n=\infty} \frac{\sum_{\mathrm{I}}^{n} b_{n}}{\lg \varphi_{n}}=\mathrm{I} .
$$

Questa proposizione è una conseguenza ummediara della formula $(7)$.

In particolare: facendo $\varphi_{n}=n$, si ha la nota proprietà della serie armonica, di divergere come $\lg n$.

II. Se la serie a termini posttiv $\sum \frac{\mathrm{I}}{u_{n}}$ è divergente e soddusfa la condizione $\lim _{n=\infty} \frac{I}{u_{n}}=0$, se si indica la somma dei suoi primi $n$ termint col simbolo $\sigma_{n}$ e si forma la serie $\sum \frac{\mathrm{I}}{u_{n} \sigma_{n}}$, questa si comporta assintoticamente come $\lg \sigma_{n}$.

Più precisamente si ha

$$
\lim _{n=\infty} \frac{\sum_{r=1}^{n} \frac{I}{u_{r} \sigma_{r}}}{\lg \sigma_{n}}=\mathrm{I} .
$$

Si deduce ciò dalla proposizione precedente col fare $\varphi_{n}=\sigma_{n}$.

La successiva applicazione $d_{1}$ cotesio risultamento conduce alla co- 
struzione della scala di serie divergenti

(I7) $\sum \frac{\mathrm{I}}{u_{n}}, \quad \sum \frac{\mathrm{I}}{u_{n} \sigma_{n}}, \quad \sum \frac{\mathrm{I}}{u_{n} \sigma_{n} \lg \sigma_{n}}, \quad \sum \frac{\mathrm{I}}{u_{n} \sigma_{n} \lg \sigma_{n} \lg \lg \sigma_{n}}, \ldots$

con la proprietà che la somma der prumi u termini do una qualunque di esse serne, tende all'infinito con rapidita eguale a quella del logaritno della somma analoga, nella serte precedente.

Le proposizioni precedenti sono note da gran tempo, si trovano infatti nella memoria del DrNI “Sulle serie a termmi posttvv» stampata negli “Annali delle Università Toscane » del 1867, e sono qui recate unicamente per dare esempio della brevità del metodo.

Tenendo conto della proposizione:

serie :

Se la serie $\sum_{i}^{\infty} \frac{\mathrm{r}}{u_{n}}$ è divergente, se $p$ indica un numero positivo, la

$$
\sum_{\mathrm{I}}^{\infty} b_{n}=\sum_{\mathrm{I}}^{\infty} \frac{\mathrm{I}}{u_{n} \sigma_{n}^{\mathrm{I}+\rho}}, \quad\left(\sigma_{n}=\sum_{\mathrm{I}}^{n} \frac{\mathrm{I}}{u_{n}}\right),
$$

è convergente ${ }^{*}$ );

e giovandoci della prop. II, formeremo la scala di serie convergentı:

$$
\text { (I9) } \frac{\mathrm{I}}{u_{n} \sigma^{\mathrm{I}+\rho}}, \quad \frac{\mathrm{I}}{u_{n} \sigma_{n}\left(\lg \sigma_{n}\right)^{\mathrm{I}+\rho}}, \quad \frac{\mathrm{I}}{u_{n} \sigma_{n} \lg \sigma_{n}\left(\lg \lg \sigma_{n}\right)^{\mathrm{I}+\rho}}, \ldots
$$

Donde i soliti cnterî, detti logaritmic, di convergenza per la serie a termini positivi ${ }^{* *}$ ).

III. Se la scrue $\sum_{\bar{I}}^{m} \frac{I}{u_{n}}$ diverge ed è $\lim _{n=\infty} \frac{\mathrm{I}}{u_{n}}=0$, la serie $\sum_{1}^{\infty} \frac{\sigma_{n}}{u_{n}}$ diveroe con rapidita eguale a quella diulla valuble $\frac{\sigma_{n}^{2}}{2}$.

Infatri si ha:

$$
\lim _{n=\infty} \frac{\sum_{1}^{n} \frac{\sigma_{1}}{u_{r}}}{\frac{\sigma_{n}^{2}}{2}}=\lim _{n=\infty} \frac{\frac{\sigma_{n}}{u_{n}}}{\frac{\sigma_{n}^{2}}{2}-\frac{\sigma_{n-1}^{2}}{2}}=\lim _{n=\infty} \frac{\sigma_{n}}{\sigma_{n}-\frac{\mathrm{I}}{2 u_{n}}}=\mathrm{I} .
$$

In particolare: la serie $\sum_{\mathrm{I}}^{\infty} \frac{\lg n}{n}$ diverge come $\frac{\mathrm{I}}{2}(\lg n)^{2}$, cloè come $\lg \left(\frac{\lg n}{n^{2}}\right) ;$ la serie $\sum \frac{\lg \sigma_{n}}{u_{n} \sigma_{n}}$ diverge come $\lg \left(\frac{\lg \sigma_{n}}{\sigma_{n}}\right)$.

") Cf. Dini, loc. c1t., $\$ 6$.

**) DiNi, loc. cit., $\$ 8$. 


\section{$\$$ III.}

ro. Abbiamo visto al $\mathrm{n}^{\circ} \mathrm{s}$ che l'ordine di infinito del prodotto $\prod_{\mathrm{r}}^{\infty}\left(\mathrm{I}+a_{n}\right)$ è minore od uguale a quello della espressione $e^{\stackrel{\infty}{\sum^{\mathrm{s}}} a_{n}}$. L'eguaglianza do quegh ordin do infinito pù stcitramente affermarsi quando la serie $\sum a_{n}^{2}$ sia convergente.

Si consideri infatti la serie:

(20) $a_{1}-\lg \left(\mathrm{I}+a_{1}\right)+a_{2}-\lg \left(\mathrm{I}+a_{2}\right)+a_{3}-\lg \left(\mathrm{I}+a_{3}\right)+\cdots$

Se poniamo

$$
S_{n}=C_{2 n}=\sum_{\mathrm{I}}^{n}\left[a_{r}-\lg \left(\mathrm{I}+a_{r}\right)\right], \quad S_{n}^{\prime}=\sum_{\mathrm{I}}^{n} a_{r}^{2},
$$

abbiamo :

$$
\lim _{n=\infty} \frac{S_{n}}{S_{n}^{\prime}}=\lim _{n=\infty} \frac{a_{n}-\lg \left(1+a_{n}\right)}{a_{n}^{2}} .
$$

Ora si ha:

$$
\lim _{x=0} \frac{x-\lg (1+x)}{x^{2}}=\lim _{x=0} \frac{1-\frac{1}{1+x}}{2 x}=\lim _{x=0} \frac{\frac{1}{(I+x)^{2}}}{2}=\frac{1}{2}:
$$

avremo anche perciò :

$$
\lim _{n=\infty} \frac{S_{n}}{S_{n}^{\prime}}=\lim _{n=\infty} \frac{a_{n}-\lg \left(\mathrm{I}+a_{n}\right)}{a_{n}^{2}}=\frac{\mathrm{I}}{2} ;
$$

d'onde si vede che le somme $C_{2 n}$ di un numero pari d1 termini, della serie (20) convergono verso un limite finito $C$ quando la serie $\sum a_{n}^{2}$ sia convergente.

Le somme $C_{2 n+1}$ di un numero disparı di termini, differiscono dalle corrispondenti somme $C_{2 n}$ per le quantità infinitesime $a_{n+1}$, e tendono perciò al medesimo limite.

La costante positiva $C$ somma della serie (20), nel caso particolare di $a_{n}=\frac{\mathrm{I}}{n}$, assume il valore $0,577215664 \ldots$ ed ha nome da EULERO ${ }^{*}$ ).

Se scriviamo la somma $C_{2 n}$ dei primi $2 n$ termini della serie (20)

*) Cf. Cesàro, loc. cit. pag. 147. 
sotto la forma :

(2I) $C_{2 n}=a_{1}+a_{2}+\cdots+a_{n}-\lg \left(\mathrm{I}+a_{1}\right)\left(\mathrm{I}+a_{2}\right) \ldots\left(\mathrm{I}+a_{n}\right)=A_{n}-\lg \varphi_{n}$ abbiamo

$$
\lim _{n=\infty}\left(A_{n}-\lg \varphi_{n}\right)=C
$$

e perciò :

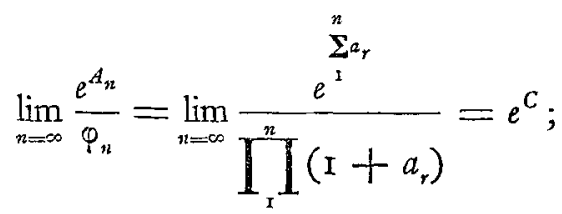

e ciò prova l'eguaglianza negli ordini di infinito delle variabili $e^{x}$, $\prod_{\mathrm{r}}^{n}\left(\mathrm{I}+a_{r}\right)$.

I I. Paragoniamo le serie a termini positivi

$$
\begin{gathered}
\sum_{1}^{\infty}-\left[a_{n}+\lg \left(\mathrm{I}-a_{n}\right)\right] \\
\sum_{\mathrm{r}}^{\infty}\left[a_{n}-\lg \left(\mathbf{1}+a_{n}\right)\right] .
\end{gathered}
$$

Facendo il rapporto dei termini corrispondenti :

e considerando che :

$$
\frac{-a_{n}-\lg \left(1-a_{n}\right)}{a_{n}-\lg \left(1+a_{n}\right)}
$$

$$
\lim _{x \rightarrow 0} \frac{-x-\lg (1-x)}{x-\lg (1+x)}=\lim _{x=0} \frac{-I+\frac{1}{I-x}}{I-\frac{1}{I+x}}=\lim _{x=0} \frac{\frac{1}{(I-x)^{2}}}{\frac{I}{(I+x)^{2}}}=\mathrm{I},
$$

e che la variabile $a_{n}$ è supposta infinitesima per $n=\infty$, si ricava subito :

$$
\lim _{n=\infty} \frac{-a_{n}-\lg \left(\mathrm{I}-a_{n}\right)}{a_{n}-\lg \left(\mathrm{I}+a_{n}\right)}=\mathrm{I} \text {. }
$$

Le due serie (23), (20) percio convergono, o divergono insieme.

Chiamando con $C_{n}^{\prime}$ la somma dei primi $n$ termini della (23), avremo

$$
C_{n}^{\prime}=\sum_{r=1}^{n}\left(-a_{r}\right)-\lg \prod_{r=1}^{n}\left(1-a_{r}\right)>0,
$$

cioè : 
(24)

$$
\frac{e^{-\sum_{\mathrm{r}}^{n} a_{r}}}{\prod_{r=1}^{n}\left(\mathrm{I}-a_{r}\right)}=e^{C_{n}^{\prime}}>\mathrm{I} .
$$

Nel caso che la serie (23) converga, cio che in particolare ba luogo quando la serie $\sum a_{n}^{2}$ è convergente, indicandone la somma con $C^{\prime}$, si ba:

$$
\lim _{n=\infty} \frac{e^{-\sum_{I}^{n} a_{r}}}{\prod_{1}^{n} I\left(I-a_{r}\right)}=e^{C^{\prime}} .
$$

Da cui si scorge che se la serie $\sum a_{r}^{2}$ converge e la serie $\sum a_{r}$ diverge, le variabili $e^{-\sum^{n} a_{r}}, \prod_{1}^{n}\left(1-a_{r}\right)$ banno lo stesso ordine do infunitesimo.

12. Le serie $\sum a_{r}^{2}, \sum b_{r}^{2}$, dove le $a_{r}, b_{r}$ sono variabili positive, convergano entrambe : convergeranno anche le serie $\sum\left[a_{r}-\lg \left(\mathrm{r}+a_{r}\right)\right]$ $\sum-\left[b_{r}+\lg \left(\mathrm{I}-b_{r}\right)\right]$.

Indicando con $C_{a}, C_{b}^{\prime}$ le somme di queste serie, avremo:

$\lim _{n=\infty}\left[\sum_{i}^{n} a_{r}-\lg \prod_{1}^{n}\left[\left(\mathrm{I}+a_{r}\right)+\sum_{i}^{n}\left(-b_{r}\right)-\lg \prod_{x}^{n}\left(1-b_{r}\right)\right]=C_{a}+C_{b}^{\prime} ;\right.$

da cui

$$
\lim _{n=\infty} \frac{e^{\sum_{x}^{n}\left(a_{r}-b_{r}\right)}}{\prod_{\mathrm{I}}^{n}\left(\mathrm{I}+a_{r}\right)\left(\mathrm{I}-b_{n}\right)}=e^{C_{n}+C_{b}^{\prime}} .
$$

I3. Sia $c_{r}$ una variabile reale infinitesima per $r=\infty$.

Consideriamo la serie

$$
\sum_{T}^{\infty}\left[c_{r}-\lg \left(x+c_{r}\right)\right]
$$

Potremo supporre tutte le $\left|c_{r}\right|$ minori di $\mathrm{I}$, ed allora tanto $\mathrm{i}$ termini corrispondenti a valori positivi delle $c_{r}$, quanto quelli corrispondenti a valori negativi sono positivi, e la serie (26) è a termini positivi. 
Ponendo

$$
C_{n, c}=\sum_{\Gamma}^{n}\left[c_{r}-\lg \left(\mathrm{I}+c_{r}\right)\right]
$$

ne verrà, per ogni valore di $n$ :

(27)

$$
\frac{e^{\sum_{I}^{n} c_{r}}}{\prod_{I}^{n}\left(I+c_{r}\right)}=e^{C_{n, c}} .
$$

Considerando ora che la serie (26) converge sempre, o diverge, assolutamente, si scorge che la variabile $C_{n, c}$ non cambia il suo comportamento assintotico qualunque inversione si faccia nell'ordine delle $c_{r}$.

La formula (27) ci offre dunque un mezzo semplicissimo per lo studio della convergenza condizionata del prodotto infinito $\prod_{1}^{\infty}\left(1+c_{r}\right)$.

I4. Senza entrare ora nei dettagli della discussione di quella formula mi limiterò ad osservare che, tutti $i$ teoremi dai vari autori trovati su questo argomento, se ne derivano con la massima facilità.

Taccio del teorema sulla convergenza assoluta, che ne è conseguenza immediata.

Supponiamo che la serie $\sum c_{r}^{2}$ sia convergente.

Convergeranno entrambe le serie $\sum a_{r}^{2}, \sum b_{r}^{2}$, dove $a_{r}$ e $b_{r}$ indicano $i$ termini positivi ed $\mathrm{i}$ valori assoluti dei termini negativi delle $c_{r}$.

Convergono quindi le serie $\sum\left[a_{r}-\lg \left(\mathrm{I}+a_{r}\right)\right], \sum\left[-b_{r}-\lg \left(\mathrm{I}-b_{r}\right)\right]$, e la serie (26) converge assolutamente.

La relazione

$$
\lim _{n=\infty} \frac{e^{\sum^{n} c_{r}}}{\prod_{r}^{n} I\left(I+c_{r}\right)}=\lim _{n=\infty} e^{c_{n, c}}=e^{C}
$$

vale perciò indipendentemente dall'ordine delle $c_{r}$.

Se la serie $\sum_{\mathrm{I}}^{\infty} c_{r}^{2}$ converge ed, in uno speciale ordinamento, la $\sum_{\mathrm{r}}^{\infty} c_{r}$ converge, in quello specale ordinamento converge anche il prodotto $\prod_{\mathrm{x}}^{\infty}\left(\mathrm{I}+c_{r}\right)$; 
e soddisfa la relazione

$$
\prod_{I}^{\infty}\left(I+c_{r}\right)=e^{\sum_{1}^{\infty} c_{r}-C} .
$$

Se, ordinando diversamente $\mathrm{i}$ termini $c$, la serte $\sum_{\mathrm{r}}^{\infty} c_{r}$ tende a $+\infty(-\infty)$, il prodotto $\prod_{\mathrm{t}}^{\infty}\left(\mathrm{I}+c_{r}\right) \dot{e}$ infinto (infinitesimo) delio stesso ordine della espressione $e^{\sum_{\Sigma}^{\infty} c_{r}}$.

I5. La serie $\sum c_{r}^{2}$ sia divergente; divergerd assolutamente anche la serie a termini positivi (26) e la relazione:

$$
\lim _{n=\infty} \frac{e^{\sum_{x}^{n} c_{r}}}{\prod_{1}^{n}\left(I+c_{r}\right)}=+\infty
$$

sard verificata per qualunque ordinamento delle $c_{r}$.

Se per un particolare ordine delle $c_{r}$ la serie $\sum_{\delta}^{\infty} c_{r}$ rusulterà convergente, $t$ corrispondente prodotto $\prod_{\mathrm{r}}^{\infty}\left(\mathrm{I}+c_{r}\right)$ sarà infintesimo di ordine eguale a quello della espressione $e^{-\sum_{x}^{\infty} c_{\tau}^{2}}$.

\I.

I6. Sieno $a_{r}, b_{r}$ variabili positive.

Poniamo

(30) $\left\{\begin{array}{l}A_{n}=a_{\mathrm{I}}+a_{2}+\cdots+a_{n}, \quad \varphi_{n}=\left(\mathrm{I}+a_{\mathrm{r}}\right)\left(\mathrm{I}+a_{2}\right) \cdots\left(\mathrm{I}+a_{n}\right) \\ B_{n}=b_{1}+b_{2}+\cdots+b_{n}, \quad \psi_{n}=\left(\mathrm{I}+b_{\mathrm{I}}\right)\left(\mathrm{I}+b_{2}\right) \cdots\left(\mathrm{I}+b_{n}\right) \\ C_{n, a}=a_{1}-\lg \left(\mathrm{I}+a_{1}\right)+a_{2}-\lg \left(\mathrm{I}+a_{2}\right)+\cdots+a_{n}-\lg \left(\mathrm{I}+a_{n}\right)=A_{n}-\lg \varphi_{n} \\ C_{n, b}=b_{1}-\lg \left(\mathrm{I}+b_{1}\right)+b_{2}-\lg \left(\mathrm{I}+b_{2}\right)+\cdots+b_{n}-\lg \left(\mathrm{I}+b_{n}\right)=B_{n}-\lg \psi_{n} .\end{array}\right.$

Considerando che la funzione :

$$
y=x-\lg (\mathrm{I}+x)
$$

è, nel tratto $(0, \ldots+\infty)$, continuamente crescente, si vede che, per 
ogni valore di $n$ e qualunque sia il comportamento assintotico della variabile positiva $a_{n}$,

$$
A_{n}>\lg \varphi_{n}, \quad e^{\sum_{r=1}^{n} a_{r}}>\prod_{r=1}^{n}\left(1+a_{r}\right) .
$$

Donde in particolare risulta che l'ordine di infinito del prodotto $\prod_{1}^{\infty}\left(1+a_{r}\right)$

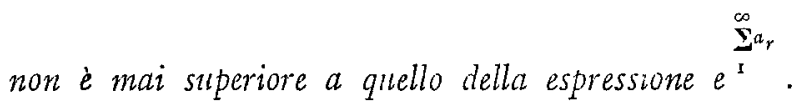

In secondo luogo supponiamo:

avremo

$$
a_{r} \supseteq b_{r} \quad(r=\mathrm{I}, 2,3, \ldots)
$$

cioè

$$
A_{n}-\lg \varphi_{n} \gg B_{n}-\lg \psi_{n}
$$

$$
C_{n, a} \supseteq C_{n, b} \text {. }
$$

Dato adunque che le serie $\sum_{i}^{\infty}\left[a_{r}-\lg \left(\mathrm{I}+a_{r}\right)\right], \sum_{\mathbf{r}}^{\infty}\left[\left(b_{1}-\lg \left(\mathbf{I}+b_{r}\right)\right]\right.$ convergano verso limiti finiti $C_{a}, C_{b}$, avreno

da cui :

$$
C_{a} \gg C_{b}
$$

La costante $C_{a}$ somma della serie $\sum\left[a_{r}-\lg \left(\mathrm{I}+a_{r}\right)\right]$ è tanto pù piccola quanto piu rapida è la convergenza allo zero della variabile $a_{r}$. Considerando ora che per la serie divergente $\sum \frac{1}{n}$ si ha $C=0,577 \ldots$, si scorge che, per serie $\sum a_{n}$ convergenti, si ottengono costanti $C_{a}$ abbastanza piccole (minori delle somme $\frac{1}{2} \sum_{1}^{\infty} a_{n}^{2}$ ) e che, per ottenere una approssimazione in eccesso del valore del prodotto infinito convergente $\prod_{\mathbf{I}}^{\infty}\left(\mathrm{I}+a_{r}\right)$, si può calcolare la espressione $e^{\sum^{a} a_{r}}$.

I7. Conservando le notazioni (30), donde in particolare si deriva :

$$
\text { (33) } \sum_{1}^{n}\left[a_{r}+b_{r}-\lg \left(\mathrm{I}+a_{r}\right)\left(\mathrm{I}+b_{1}\right)\right]=A_{n}+B_{n}-\lg \varphi_{n} \downarrow_{1 n}=C_{n, a}-C_{n, b} \text {, }
$$

poniamo inoltre :

$$
\sum_{1}^{n}\left[a_{r}+b_{r}-\lg \left(\mathrm{I}+a_{r}+b_{r}\right)\right]=C_{n, a+b} .
$$


Osservando che per $a_{r}>0, b,>0$, si ha:

e che perciò :

$$
\mathrm{I}+a_{r}+b_{r}<\left(\mathrm{I}+a_{r}\right)\left(\mathrm{I}+b_{r}\right)
$$

(35) $a_{r}+b_{r}-\lg \left(\mathrm{I}+a_{r}+b_{r}\right)>a_{r}+b_{r}-\lg \left(\mathrm{I}+a_{r}\right)\left(\mathrm{I}+b_{r}\right)$, ricordando inoltre che la funzione $x-\lg (s+x)$ per valor1 positivi della $x$ è sempre crescente, abbiamo

$a_{r}+b_{r}+a_{r} b_{r}-\operatorname{lo}\left(\mathrm{I}+a_{r}\right)\left(\mathrm{I}+b_{r}\right)>a_{r}+b_{r}-l_{r}\left(\mathrm{I}+a_{1}+b_{r}\right)$

da cui$$
>a_{r}+b_{r}-\lg \left(\mathrm{I}+a_{r}\right)\left(\mathrm{I}+b_{r}\right)
$$

$$
C_{n, a}+C_{n, b}+\sum_{i}^{n} a_{r} b_{r}>C_{n, a+b}>C_{n, a}+C_{n, b} .
$$

Ne concludamo che: Se esistono, determinali e finth, $i$ lannti per $n=\infty$ delle $C_{n, a}, C_{n, b}$, e se la serte $\sum_{i}^{\infty} a_{n} b_{n}$ è convergente, estste anche limute finuo per la variabile $C_{n, a+b}$ e soddisfa la hinitazione:

$$
\lim _{n=\infty}\left(C_{n, a}+C_{n, b}\right)<\lim _{n=\infty} C_{n, n+b}<\lim _{n=\infty}\left(C_{n, a}+C_{n, b}\right)+\sum_{i}^{\infty} a_{r} b_{r} .
$$

I8. Supponiamo ora che

$$
a_{r}+b,<\mathrm{I} \quad(r=\mathrm{I}, 2,3 \ldots) .
$$

Facilmente verificheremo che :

(37) $0<\lg (\mathrm{I}+a)\left(\mathrm{I}+b_{r}\right)-\lg \left(\mathrm{I}+a_{1}+b_{1}\right)<a_{r}+b_{r}-\lg \left(\mathrm{I}+a_{r}\right)\left(\mathrm{I}+b_{r}\right)$,

da cui

$$
0<\lg \prod_{1}^{n}\left(\mathrm{I}+a_{r}\right)\left(\mathrm{I}+b_{r}\right)-\lg \prod_{\mathrm{r}}^{n}\left(\mathrm{I}+a_{1}+b_{r}\right)<C_{n, a}+C_{u, b},
$$

infine

$$
\mathrm{I}<\frac{\prod_{\mathrm{r}}^{n}\left(\mathrm{I}+a_{r}\right)\left(\mathrm{I}+b_{1}\right)}{\prod_{\mathrm{r}}^{n}\left(\mathrm{I}+a_{1}+b_{r}\right)}<e^{c_{n, t}+C_{n, b}} .
$$

In particolare: se le serie $C_{n, n}, C_{n, b}$ convergono, ed a due prodotte infinti $\prod_{1}^{\infty}\left(\mathrm{I}+a_{n}\right)\left(\mathrm{I}+b_{n}\right), \prod_{1}^{\infty}\left(\mathrm{I}+a_{n}+b_{n}\right)$ sono divergenit, essi hanno lo stesso ardine de infinito; se invec? convergono, sono fra loro nella relazione: 
(40) $\prod_{\mathrm{I}}^{\infty}\left(\mathrm{I}+a_{r}+b_{r}\right)<\prod_{\mathrm{I}}^{\infty}\left(\mathrm{I}+a_{n}\right)\left(\mathrm{I}+b_{n}\right)<\prod_{\mathrm{I}}^{\infty}\left(\mathrm{I}+a_{r}+b_{r}\right) \cdot e^{c_{a}+c_{b}}$.

Se scriviamo la (39) sotto la forma

$$
\mathrm{I}<\prod_{\mathrm{I}}^{n}\left(\mathrm{I}+\frac{a_{r} b_{r}}{\mathrm{I}+a_{r}+b_{r}}\right)<e^{C_{n, a}+C_{n, b}}
$$

scorgiamo che: se $l$ serie $C_{n, i}, C_{n, b}$ sono convergenti, converge 1 prodotto infinito $\prod_{1}^{\infty}\left(\mathrm{I}+\frac{a_{n} b_{n}}{\mathrm{I}+a_{n}+b_{n}}\right)$ : converge dunque anche la serie :

$$
\sum_{\mathbf{1}}^{\infty} \frac{a_{n} b_{n}}{\mathrm{I}+a_{n}+b_{n}}
$$

ed infine converge la serve $\sum_{r}^{\infty} a_{n} b_{n}$, i cui termini banno a quelli della serie precedente rapports tendenti al limite $I$; ma dalla formola (36) deduciamo che in allora converge anche la $C_{n, a+1}$; in fine concluderemo:

Se le serue $\sum_{\mathrm{I}}^{\infty}\left[a_{n}-\lg \left(\mathrm{I}+a_{n}\right)\right], \sum_{\mathrm{I}}^{\infty}\left[\left(b_{n}-\lg \left(\mathrm{I}+b_{n}\right)\right]\right.$ sono entrambe convergenti, convergono anche le serve:

$$
\sum_{i}^{\infty} a_{n} b_{n}, \quad \sum_{1}^{\infty}\left[a_{n}+b_{n}-\lg \left(\mathrm{I}+a_{n}+b_{n}\right)\right]
$$

19. Scrivendo la formula (37) sotto la forma

$$
\begin{gathered}
a_{r}+b_{r}-\lg \left(\mathrm{x}+a_{r}+b_{r}\right)-\left[a_{r}+b_{r}-\lg \left(1+a_{r}\right)\left(\mathrm{I}+b_{r}\right)\right] \\
<a_{r}+b_{r}-\lg \left(\mathrm{x}+a_{r}\right)\left(\mathrm{x}+b_{r}\right)
\end{gathered}
$$

e sommando da $r==I$ ad $n$, si ha :

cioè

$$
C_{n, a+b}-\left(C_{n, a}+C_{n, b}\right)<C_{n, a}+C_{n, b},
$$

$$
C_{n, a}+C_{n, b}<C_{n, a+b}<2\left(C_{n, a}+C_{n, b}\right) \text {. }
$$

Osserviamo ora che per $x>0$, si ha

$$
x-\lg (1+x)<\frac{x^{2}}{2},
$$

d'onde si ricava:

ed avremo

$$
C_{n, a}+C_{n, b}<\sum_{i}^{n} \frac{a_{1}^{2}+b_{1}^{2}}{2}
$$

$$
C_{n, a+b}<\sum_{I}^{n}\left(a_{r}^{2}+b_{r}^{2}\right)
$$


da cui, ricordando la (34), dedurremo:

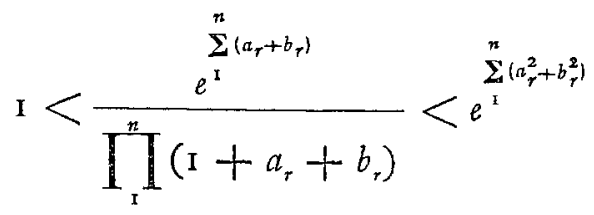

In particolare, se la somma $\sum\left(a_{r}+b_{r}\right)$ è convergente, si ha la limitazione

$$
e^{\sum_{1}^{\infty}\left(a_{r}+b_{r}-a_{r}^{2}-b_{r}^{2}\right)}>\sum_{1}^{\infty}\left(1+a_{r}+b_{r}\right)<e^{\sum_{1}^{\infty}\left(a_{r}+b\right)} .
$$

20. Dalla formula (42), facendo $b=a$, si ricava.

$$
{ }_{2} C_{n, a}<C_{n, 2 a}<{ }_{4} C_{n, a} \text {. }
$$

Di pol, facendo $b=2 a$ :

$$
{ }_{3} C_{n, a}<C_{n, 3 a}<\text { го } C_{n, a} \text {. }
$$
ferenze :

In generale, indicando con $P_{p}$ l'integrale della equazione alle dif(45)

$$
f_{p}-2 f_{p-5}-2=0
$$

che è determinato dal valore iniziale $P_{\mathrm{I}}=\mathrm{I}$, si ha

$$
p C_{n, a}<C_{n, p a}<P_{p} C_{n, a} .
$$

I numeri $P_{p}$ sono tuts finiti, epperò, in particolare, abbiamo:

Se la serie $\sum_{\mathrm{r}}^{\infty}\left[a_{r}-\lg \left(\mathrm{I}+a_{r}\right)\right]$ converge, ed è $p$ un numero posttivo, intero, qualunque, anche la serie

$$
\sum_{1}^{\infty}\left[p a_{r}-\lg \left(1+p a_{r}\right)\right]
$$

¿ convergente; $e$, se si incomincia a sommare da un indice abbastanza elevato perche sia sempre par< $\mathrm{I}_{\text {, si }}$ ba:

(47) $\left\{\begin{array}{c}\sum_{i}^{\infty}\left[p a_{r}-\lg \left(1+p a_{r}\right)\right]=\theta \sum_{1}^{\infty}\left[a_{r}-\lg \left(1+a_{r}\right)\right] \\ p<\theta<P_{p} .\end{array}\right.$

2I. Tale risultamento si estende senza difficoltà al caso in cui $p$ rappresenti una costante positiva qualunque, e, tenendo conto, dei risultamenti ottenuti al $\$$ precedente, anche al caso di costanti negative. 
Vale un risultamento analogo anche nel caso che $p$ sia una quantità variabile ed abbia limitı superiore ed inferiore $d 1$ indeterminazione finiti.

In particolare potremo enunciare la proposizione seguente:

Se la variabule $b_{n}$ ba limute supertore di indeterminazione $L$, ed $\dot{e} p$ Il massimo intero contento $i n L$; se $1 !$ silo limite inferiore di indeterminazione è un mumero $l \searrow I$, se il prodotto $a_{n} b_{n}$, al pari della $a_{n}$, $\dot{e}$ sempre minore della unità, si ba:

$$
\left\{\begin{array}{c}
\sum_{1}^{n}\left[a_{r} b_{r}-\lg \left(\mathrm{I}+a_{r} b_{r}\right)\right]=\theta_{n} \sum_{i}^{n}\left[a_{r}-\lg \left(\mathrm{I}+a_{r}\right)\right] \\
l<\theta_{n}<P_{p} .
\end{array}\right.
$$

Se la vartabile $b_{n}$ ba limute inferiore $d_{l}$ indeterminazione $l>1$, anche se non si possa accertare che tutte le $a_{n} b_{n}$ sieno minori di $\mathrm{I}$, si potrà sempre affermare che:

$$
\sum_{1}^{n}\left[a_{r} b_{r}-\lg \left(1+a_{r} b_{r}\right)\right]>l \sum_{1}^{n}\left[a_{r}-\lg \left(\mathbf{1}+a_{r}\right)\right]
$$

22. Se le serie $\sum_{1}^{\infty}\left[a_{r}^{\prime}-\lg \left(\mathrm{I}+a_{r}^{\prime}\right], \sum\left[a_{r}^{\prime \prime}-\lg \left(\mathrm{r}+a_{r}^{\prime \prime}\right)\right], \ldots\right.$ sono tutte convergenti, se le varabuli fosttve $b_{r}^{\prime}, b_{r}^{\prime \prime}, \ldots$ banno limati superiori in indelermmazione finth, converge anche la serue

$$
\sum\left[a_{r}^{\prime} b_{r}^{\prime}+a_{r}^{\prime \prime} b_{r}^{\prime \prime}+\cdots-\lg \left(\mathrm{I}+a_{r}^{\prime} b_{r}^{\prime}+a_{r}^{\prime \prime} b_{r}^{\prime \prime}+\cdots\right)\right] \text {. }
$$

23. Supponiamo che la serie $\sum_{i}^{\infty}\left[a_{n}-\lg \left(1+a_{n}\right)\right]$ sia divergente.

In tale ipotest, esrendo $\alpha$ una costante positiva (o negativa) qualunque, potremo scrivere:

$$
\lim _{n=\infty} \frac{\sum_{i}^{n}\left[\alpha a_{r}-\lg \left(1+\alpha a_{r}\right)\right]}{\sum_{i}^{n}\left[\left(a_{r}-\lg \left(1+a_{r}\right)\right]\right.}=\lim _{n=\infty} \frac{\alpha a_{r}-\lg \left(1+\alpha a_{r}\right)}{a_{r}-\lg \left(1+a_{r}\right)}
$$

ed, analogamente a quanto si fece $a l n^{\circ}$ I I, concluderemo:

$$
\lim _{n=\infty} \frac{\sum_{1}^{n}\left[\alpha a_{r}-\lg \left(1+\alpha a_{r}\right)\right]}{\sum_{r}^{n}\left[\left(a_{r}-\lg \left(1+a_{r}\right)\right]\right.}=\alpha^{2} .
$$


Di qui facilmente si deduce

$$
\lim _{n=\infty} \frac{\sum_{I}^{n}\left[\alpha a_{r}-\lg \left(\mathrm{I}+\alpha a_{r}\right)\right]-\alpha \sum_{I}^{n}\left[\left(a_{r}-\lg \left(\mathrm{I}+a_{r}\right)\right]\right.}{\alpha \sum_{1}^{n}\left[a_{r}-\lg \left(\mathrm{I}+a_{r}\right)\right]}=\alpha-\mathrm{I},
$$

cioè :

$$
\lim _{n=\infty}-\lg \frac{\prod_{1}^{n}\left(\mathrm{I}+\alpha a_{r}\right)}{\left[\prod_{1}^{n}\left[\left(\mathrm{I}+a_{r}\right)\right]^{\alpha}\right.} \cdot \frac{\mathrm{I}}{\alpha C_{n, a}}=\alpha-\mathrm{I},
$$

che scriveremo:

$$
-\lg \frac{\prod_{1}^{n}\left(\mathrm{I}+\alpha a_{r}\right)}{\left[\prod_{\mathrm{r}}^{n}\left(\mathrm{I}+a_{r}\right)\right]^{\alpha}} \cdot \frac{\mathrm{I}}{\alpha C_{n, n}}=\alpha-\mathrm{I}+\varepsilon_{n}, \quad \lim _{n=1} \varepsilon_{n}=0,
$$

cioè

$$
\frac{\prod_{1}^{n}\left(\mathrm{I}+\alpha a_{r}\right)}{\left[\prod_{\mathrm{I}}^{n}\left[\left(\mathrm{I}+a_{r}\right)\right]^{\alpha}\right.}=e^{-\alpha C_{n, \alpha}\left(\alpha-\mathrm{I}+\varepsilon_{n}\right)}, \quad \lim _{n=\infty} \varepsilon_{n}=0
$$

infine :

$(s \mathrm{I}) \prod_{\mathrm{I}}^{n}\left(\mathrm{I}+\alpha a_{r}\right)=\left[\prod_{\mathrm{I}}^{n}\left(\mathrm{I}+a_{r}\right)\right]^{\alpha} \cdot e^{-\alpha C_{n, a}\left(\alpha-1+\varepsilon_{n}\right)}, \quad \lim _{n=\infty} \varepsilon_{n}=0$.

Ricordando ora che $C_{n, a}=\sum_{\mathrm{I}}^{n} a_{r}-\lg \prod_{\mathrm{I}}^{n}\left(1+a_{r}\right)$, e che, nella ipotesi $\lim _{n=\infty} a_{n}=0$, da cui non ci siamo dipartiti, si ha

avremo

$$
\lim _{n=\infty} \frac{\sum_{\mathrm{T}}^{n} a_{r}}{\lg \prod_{1}^{n}\left(1+a_{r}\right)}=\mathrm{I}
$$

$\frac{C_{n, a}}{\lg \prod_{1}^{n}\left(1+a_{r}\right)}=\frac{\sum_{1}^{n} a_{r}}{\lg \prod_{1}^{n}\left(1+a_{r}\right)}-\mathrm{I}=\sigma_{n}, \quad \lim _{n=\infty} \sigma_{n}=0, \quad \sigma_{n}>0$ dunque 


$$
\begin{aligned}
& C_{n, a}=\lg \left[\prod_{\mathrm{s}}^{n}\left(\mathrm{I}+a_{r}\right)\right]^{\sigma_{n}}, \quad \lim _{n=\infty} \sigma_{n}=0, \quad \sigma_{n}>0 \\
& e^{C_{n, a}}=\left[\prod_{\mathrm{x}}^{n}\left(\mathrm{I}+a_{r}\right)\right]^{\sigma_{n}} ; \quad\left\{\begin{array}{c}
e^{\alpha C_{n, a}\left(\alpha_{\mathrm{I}}+\varepsilon_{n}\right)}=\left[\prod_{\mathrm{I}}^{n}\left(\mathrm{I}+a_{n}\right)\right]^{\delta_{n}} \\
\delta_{n}>0, \quad \lim _{n=\infty} \delta_{n}=0 .
\end{array}\right.
\end{aligned}
$$

Sostituendo nelle ( 5 I) ho

$$
\text { (52) } \prod_{\mathrm{I}}^{n}\left(\mathrm{I}+\alpha a_{r}\right)=\left[\prod_{\mathrm{I}}^{n}\left(\mathrm{I}+a_{r}\right)\right]^{\alpha-\delta_{n}}, \quad \delta_{n}>0, \quad \lim _{n=\infty} \delta_{n}=0 .
$$

Questo risultamento si può esprimere dicendo:

Se l'ordine di infinto del prodotto $\prod_{\mathrm{s}}^{\infty}\left(\mathrm{I}+a_{r}\right)$ si assume come $u$ nità, l'ordine del prodotto $\prod_{\mathrm{r}}^{\infty}\left(\mathrm{I}+\alpha a_{\mathrm{r}}\right)$ non è maggiore di $\alpha$ ed è maggiore di $\alpha-a$, a positivo qualunque.

Usando la notazione che il Borel, seguendo le idee di Cauchy, ha proposto ${ }^{*}$ ), diremo che quell'ordine è espresso dal simbolo $(\alpha)$.

24. Nel caso che la serie $\sum_{\Gamma}^{\infty}\left(a_{n}-\lg \left(1+a_{n}\right)\right.$ sia convergente, giovandoci del risultamento espresso dalla formola (47), con ragionamento analogo a quello fatto dianzi, avremo

$$
\left\{\begin{array}{c}
\lim _{n=\infty} \frac{\prod_{1}^{n}\left(\mathrm{I}+\alpha a_{r}\right)}{\left[\prod_{\mathrm{I}}^{n}\left(\mathrm{I}+a_{r}\right)\right]^{\alpha}}=e^{(\alpha-0) c_{a}} \\
\theta \text { determinato e finito, } C_{n}=\sum_{\mathrm{s}}^{\infty}\left[a_{n}-\lg \left(\mathrm{I}+a_{n}\right)\right] .
\end{array}\right.
$$

D'onde concluderemo che, preso per unità l'ordine di infinito del prodotto $\prod_{\mathrm{I}}^{\infty}\left(\mathrm{I}+a_{r}\right)$, l'ordine di infinito del prodotto $\prod_{\mathrm{I}}^{\infty}\left(\mathrm{I}+\alpha a_{r}\right) \dot{e} e-$ spresso dal numero $\alpha$, non solo secondo la definizione di CaUchY; ma

*) Cfr. E. BOREL, Leçons sur les séries à termes positifs. (Paris), pag. 36. 
anche nel senso più stretto, in cui ordinariamente è definito l'ordine di infinito.

25. Le ricerche precedenti si possono estendere a prodotti della forma $\prod_{1}^{\infty}\left(\mathrm{I}+a_{r} c_{r}\right), \prod_{1}^{\infty}\left(\mathrm{I}+a_{r}\right)$ dove $a_{r}, c_{r}$ sono variabili reali qualunque, soggette alle sole condizioni seguenti :

๔) le $a_{r}$ sono tutte di uno stesso segno e nessuna di esse è nulla,

$\beta)$ i binomı $\left(\mathrm{I}+a_{r}\right),\left(\mathrm{I}+a_{r} c_{r}\right)$, sono tutti maggiori di zero,

$\gamma)$ il prodotto infinito $\prod_{\mathrm{I}}^{\infty}\left(\mathrm{I}+a_{r}\right)$ non è convergente.

Un ragionamento analogo a quello fatto al $\mathrm{n}^{\circ} 23$, ci conduce alla ricerca del numero $\beta=\lim _{r=\infty} \frac{\lg \left(1+a_{r} c_{r}\right)}{\lg \left(1+a_{r}\right)}$.

Se, per brevtà di linguaggio, consideriamo gli infunitesimi come infiniti de ordine negativo, se per infinito principale assumiamo quello della variabile $\prod_{1}^{n}\left(\mathrm{I}+a_{n}\right)$ nella ipotesi cbe questa finisca per superare qualunque numero positivo, quello della $\left[\prod_{1}^{n}\left(\mathrm{I}+a_{r}\right)\right]^{-1}$ nel caso contrario, troveremo in ogni caso che il numero $\beta$ esprime l'ordine di infinito (al senso $d \imath$ CAUChy $) d e l$ prodotto infinito $\prod_{1}^{\infty}\left(1+a_{r} c_{r}\right)$.

26. Steno $\beta_{1}, \beta_{2}$, rispettivamente, $i$ limiti inferiore e superiore di indeterminazione della espressione $\frac{\lg \left(1+a_{r} c_{r}\right)}{\lg \left(1+a_{r}\right)}$, e sia $\varepsilon$ un numero positivo arbitrario.

La variabile $\prod_{\mathrm{I}}^{n}\left(\mathrm{I}+a_{r} c_{r}\right)$ sarà, per $n=\infty$, infinita (infinitesima), di ordine superiore a quello della variabile $\left[\prod_{\mathrm{I}}^{n}\left(\mathrm{I}+a_{r}\right)\right]^{\beta_{\mathrm{I}}-\varepsilon}$, ed inferiore a quello della variabule $\left[\prod_{1}^{n}\left(\mathrm{I}+a_{r}\right)\right]^{\beta_{1}+\varepsilon}$.

Supponiamo, per fissare le idee, che sia $\lim _{n=\infty} \prod_{1}^{n}\left(\mathrm{I}+a_{n}\right)=+\infty$.

Per la definizione di $\beta_{1}$, in corrispondenza di $\varepsilon$ determineremo 
un $N$ tale che

$$
n \geqslant N, \quad \frac{\lg \left(\mathrm{I}+a_{r} c_{r}\right)}{\lg \left(\mathrm{I}+a_{r}\right)}>\beta_{\mathrm{r}}-\varepsilon
$$

Ricordando la proprietà $\alpha$ ) dei numeri $a_{r}$, avremo:

infine :

$$
\begin{aligned}
r & \geqslant N, \quad \mathrm{I}+a_{r} c_{r}>\left(\mathrm{I}+a_{r}\right)^{\beta_{1}-\varepsilon}, \\
n>N, \quad \prod_{r=\frac{1}{N}}^{n}\left(\mathrm{I}+a_{r} c_{r}\right) & >\left[\prod_{i=\frac{N}{N}}^{n}\left(\mathrm{I}+a_{r}\right)\right]^{\beta_{3}-\varepsilon},
\end{aligned}
$$

$$
n>N, \frac{\prod_{r=1}^{n}\left(\mathrm{I}+a_{r} c_{r}\right)}{\left[\prod_{r=1}^{n}\left(\mathrm{I}+a_{r}\right)\right]^{\beta_{1}-\varepsilon}}>\frac{\prod_{r=1}^{N}\left(\mathrm{I}+a_{r} c_{r}\right)}{\left[\prod_{r=1}^{N}\left(\mathrm{I}+a_{r}\right)\right]^{\beta_{\mathrm{r}}-\varepsilon}},
$$

e ciò prova la prima parte dell'enunciato. Analoga dimostrazione si faccia per la parte che riguarda $\beta_{2}$.

Nel caso che $\beta_{1}$ risulti negativo, non potremo escludere che la variabile $\prod_{I}^{n}\left(1+a_{r} c_{r}\right)$ sia infinita di ordine negativo, cioè nel fatto infinitesima.

Il teorema e la dimostrazione valgono anche nel caso che la variabile $\prod_{1}^{n}\left(\mathrm{I}+a_{r}\right)$ tenda al limite zero e servono per determinare l'ordine di infinitesimo del prodotto $\prod_{1}^{\infty}\left(1+a_{r} c_{1}\right)$. In questo caso, a limiti inferiori $\beta_{x}$ negativi, possono effettivamente corrispondere prodotti $\prod_{\mathrm{I}}^{n}\left(\mathrm{I}+a_{r} c_{r}\right)$ infiniti per $n=\infty$.

Se è $\beta_{1}=\beta_{2}=\beta$, segue senza difficoltà la proposizione enunciata al $n^{\circ} 25$.

Se è $\beta_{2}=0$, la variabile $\prod_{1}^{n}\left(1+a_{r} c_{r}\right)$ potrà essere finita, od infinita (infinitesima) di ordine inferiore a quello di qualunque potenza positiva della $\prod_{\mathrm{l}}^{n}\left(\mathrm{I}+a_{r}\right)$.

Se è $\beta_{1}=\infty$, la $\prod_{\mathrm{I}}^{n}\left(\mathrm{I}+a_{r} c_{r}\right)$ sarà infinita (infinitesima) di ordine superiore a quello di qualunque potenza positiva della $\prod_{1}^{n}\left(1+a_{r}\right)$. 
27. Se la variabile $a_{n}$ soddisfa la relazione

$$
\lim _{n=\infty} a_{n}=0 \text {, }
$$

1 numeri $\beta_{1}, \beta_{2}, \beta$, definti ai numeri precedents si hanno cercando $i$ limiti :

$$
\beta_{3}=\liminf _{n=\infty} c_{n}, \quad \beta_{2}=\limsup _{n=\infty} c_{n}, \quad \beta=\lim _{n=\infty} c_{n} .
$$

Ciò in conseguenza del fatto:

$$
\lim _{x=0} \frac{\lg [1+x \cdot f(x)]}{\lg (1+x)}=\lim _{x=0} \frac{\frac{f(x)+x f^{\prime}(x)}{i+x f(x)}}{\frac{1}{1+x}}=\lim _{x=0} f(x) .
$$

28. Come esempio di applicazione dei criterî esposti ai $n^{1}$ precedenti, prendiamo prima in esame il prodotto $\prod_{1}^{\infty}\left(I+\frac{I}{n}\right)$.

La serie $\sum\left(\frac{\mathrm{I}}{n}-\lg \mathrm{I}+\frac{\mathrm{I}}{n}\right)$ converge (hà per somma la costante di Eulero). La serie $\sum \frac{1}{n}$ diverge come $\lg n, 1$ prodotto dato dunque diverge con la stessa rapidità del numero $n$.

Sia ora il prodotto $I\left(1+\frac{b_{n}}{n}\right), \lim _{n \in \infty} b_{n}=\beta$; divergerà esso come $n^{\beta}$.

Cioè: il prodotto $\prod_{\mathrm{I}}^{\infty}\left(\mathrm{I}+a_{n}\right)$ è infinito dell'ordine $\beta$, se la $a_{n} \dot{e}$ infinitesimo del $\mathrm{I}^{\circ}$ ordine ed ha per parte princapale $\frac{\beta}{n}$.

Risultamento trovato per altra strada nella mia Nota: Su prodotti infinitu divergenti (Rendiconti della R. Accadenia dei Lincel, $2^{\circ}$ Semestre del I90I).

Come secondo esempio consideramo il prodotto infrnito:

$$
\prod_{\mathrm{I}}^{\infty}\left(\mathrm{I}+\frac{\mathrm{I}}{n \lg n}\right) \text {. }
$$

La șerie $\sum\left[\frac{\mathrm{I}}{n \lg n}-\lg \left(\mathrm{I}+\frac{\mathrm{I}}{n \lg n}\right)\right]$ che ha $\mathrm{i}$ termini inferiori a quelli della serie considerata all'esempio precedente, sicuramente converge.

La serie $\sum \frac{\mathrm{I}}{n \lg n}$ diverge come $\lg \lg n(\$$ II, prop. II $)$; il prodotto 
proposto diverge perció come $\lg n$ : ed il prodotto

$$
\prod_{\mathrm{I}}^{\infty}\left(I+\frac{b_{n}}{n \lg n}\right), \quad \lim _{n=\infty} b_{n}=p,
$$

diverge come $(\lg n)^{p}$.

In generale: se una funzione monotona $f(x)$ soddrsfa la relazione $\frac{f(x+1)}{f(x)}=\frac{b(x)}{x \lg x}+1, \lim _{x=\infty} b(x)=p$, essa si comporta assintoticamente come la funzione $(\lg x)^{p}$. (Cfr Sur prodotti infiniti divergenti al $\mathrm{n}^{\circ}$ I3).

Prendiamo infine ad esaminare it prodotto infinito $\prod_{\mathrm{I}}^{\infty}\left(\mathrm{I}+\frac{\lg n}{n}\right)$. La serie $\sum\left[\frac{\lg n}{n}-\lg \left(I+\frac{\lg n}{n}\right)\right]$ converge, perchè è convergente la serie $\sum\left(\frac{\lg n}{n}\right)^{2}$.

La serie $\sum \frac{\lg n}{n}$ si comporta assintoticamente come la variabile $\frac{(\lg n)^{2}}{2}(\S I I$, prop. IV). Il prodotto dato ha dunque ordine di infinito eguale a quello della variabile:

Il prodotto infinito

$$
n^{\frac{\lg n}{2}}
$$

$$
\prod_{\mathrm{r}}^{\infty}\left(\mathrm{I}+b_{n} \frac{\lg n}{n}\right), \quad \lim _{n=\infty} b_{1 b}=p
$$

diverge dunque come la variabile

$$
n^{\frac{p \lg n}{2}}
$$

tale è anche la rapidità di divergenza di qualunque funzione monotona $f(x)$, la quale soddisfi la relazione

$$
\begin{gathered}
\frac{f(x+\mathrm{I})}{f(x)}=\mathrm{I}+b_{x} \frac{\lg x}{x}, \quad \lim _{x \rightarrow \infty} b_{x}=p . \\
\S \mathrm{V} .
\end{gathered}
$$

29. La nota relazione, dovuta a CAUCHY ${ }^{*}$ ), fra le serie a termini positivi sempre decrescenti $\sum_{i}^{\infty} \alpha(n)$, e gli integralı definiti improprii

*) Cfr. p. es. Goursat, Cours d'Analyse mathématique (Paris 1902), pag. 379. 
$\int_{1}^{\infty} \alpha(x) d x$, può servire a dedurre, dalle proposizioni enunciate nei $\$ \$$ precedenti, altrettante proprietà relative al comportamento assintotico delle variabili $\prod_{r=1}^{n}\left[\mathrm{I}+\alpha\left(x_{0}+r\right)\right], \int_{x_{0}}^{x_{0}+n} \alpha(x) d x$.

Nella Nota già citata Sui prodotti infiniti divergenti ho trovato direttamente le più importanti di tali proposizioni, desumendole, con metodo che ritengo nuovo, da pochi e generali princips sulla crescenza delle funzioni monotone.

30. Non è difficile dimostrare ${ }^{*}$ ) che tutte le funzion realı $f(x)$ della variabile reale $x$, monotone in un delerminato intorno dell'infinto, $e$ la cui dervoata logaritnuca è infuitesima per $x=+\infty$, soddisfano la relazione:

$$
\lim _{x=+\infty} \frac{f(x+1)}{f(x)}=\mathbf{I}
$$

e che, reciprocamente: tutte le funzion monotone $f(x)$ soddrsfacents la (54), non possono avere derivata logaritmica determinata per $x=+\infty$, se non infinitesima.

Cosi pure si può affermare che:

Se si assegnano alla classe $p^{\text {esima }}$ le funzioni monotone $f(x)$, le quali soddisfano le relazioni:

$$
\left.\lim _{x=+\infty} \frac{\lg f(x)}{x^{p}}=0, \quad \lim _{x=+\infty} \inf _{x} \cdot \frac{\lg f(x)}{x^{p-1}}>0{ }^{* *}\right),
$$

le funzion

$$
\varphi(x)=\frac{f(x+1)}{f(x)}
$$

appartengono alla classe $(p-1)^{\text {esima }}$, e rectprocamente. la forma

31. Le funzioni della prima classe possono dunque mettersi sotto

$$
f(x)=e^{x \varepsilon(x)}, \quad \lim _{x=\infty} \varepsilon(x)=0
$$

*) Cfr. Sul limite del quoziente di due funzion (Annali di Matematica, tomo VIII della serie III, pag. 273 e seguenti).

${ }^{* *}$ Scrivo: $\lim _{x=+\infty}$ inf. invece di limite inferiore di indeterminazione per $x=+\infty$. 
possono essere infinite od infinitesime, per $x=+\infty$, secondo che la $\varepsilon(x)$ è sempre positiva o sempre negatıva in un determinato intorno dell'infinito, e soddisfano tutte la condizione:

$$
\lim _{x=\infty} \frac{f(x+1)}{f(x)}=\mathbf{1} \text {. }
$$

Se rappresento con $\alpha(x)$ una funzione positiva, monotona, della variabile reale $x$, derivabile in un determinato intorno dell'infinito; con $f(x)$ l'integrale della equazione alle differenze finite

$$
f(x+1)=[1+\alpha(x)] f(x)
$$

determunato dal valore iniziale $f\left(x_{0}\right)=\mathrm{I}+\alpha\left(x_{0}\right)$, e dalla condizione di essere finita e continua insieme con le sue derivate di qualunque ordine nell'intorno $\left(x_{0}+\infty\right)$; ho le relazioni :

$$
f\left(x_{0}+n\right)=\prod_{r=0}^{n}\left[\mathrm{I}+\alpha\left(x_{0}+r\right)\right]
$$

e la $f(x)$ cosi formata sarà della prima classe, ogniqualvolta la $\alpha(x)$ sia infinitesima per $x=+\infty^{*}$ ).

Quando questo sia ammesso, la relazıone

$$
\text { (58) } \alpha(x)=\frac{f(x+1)-f(x)}{f(x)}=\frac{f^{\prime}(x+\theta)}{f(x)}=\frac{f^{\prime}(x+\theta b)}{f(x+\theta b)} \cdot \frac{f(x+\theta)}{f(x)}, \quad 0 \leqslant \theta \leqslant \mathbf{1} \text {, }
$$

permetterà di dimostrare che le funziont $\alpha(x), \frac{f^{\prime}(x)}{f(x)}$ sono insieme della classe prima, od insieme di classi superiort.

32. E facile verificare se una data funzione $\alpha(x)$ appartenga alla classe prima. Converrá esammare se una almeno delle condizıoni

$$
\lim _{x=\infty} \frac{\alpha^{\prime}}{\alpha}=0, \quad \lim _{x=\infty} \frac{\alpha(x+1)}{\alpha(x)}=1, \quad \lim _{x=\infty}[\alpha(x)]^{\frac{1}{x}}=1,
$$

sia o no soddisfatta.

Nel caso affermativo, dovendo essere anche la $\frac{f^{\prime}}{f}$ della classe prima, scriveremo :

$$
\frac{f^{\prime}}{f}=e^{-x \varepsilon(x)}, \quad \lim _{x=\infty} \varepsilon(x)=0 .
$$

*) La $f(x)$ è certamente sempre crescente; si veda al proposito: Stolz Grundzuge, vol. I, pag. 58 . Del resto, per non entrare in discussioni sottili, ammetto senz'altro che le funzioni $f(x), f^{\prime}(x), f^{\prime \prime}(x), \frac{f^{\prime}(x)}{f(x)}$, sieno tutte monotone. 
D'onde ricaveremo :

(6o) $\quad\left\{\begin{array}{l}f^{\prime}=f \cdot e^{-x \varepsilon(x)}, \quad f^{\prime \prime}=\frac{f^{\prime}}{f}\left[f^{\prime}-f \cdot\left(\varepsilon+x \varepsilon^{\prime}\right)\right] \\ \frac{f^{\prime \prime}}{f^{\prime}}=\frac{f^{\prime}}{f}-\varepsilon-\varepsilon^{\prime} x=\sigma(x), \\ \frac{f^{\prime \prime}}{f}=\frac{l^{\prime}}{f}\left(\frac{f^{\prime}}{f}-\varepsilon-x \varepsilon^{\prime}\right)=\frac{f^{\prime}}{f} \cdot \sigma(x) .\end{array}\right.$

Se ammettiamo che $\frac{f^{\prime \prime}}{f^{\prime}}$ sia, per $x=+\infty$, determinata, vediamo che $\lim _{x=+\infty} \sigma(x)=0$, e la funzione $f^{\prime \prime}$ appartiene alla prima classe, onde, se la variabile positiva $b_{x}$ ha limite superiore di indeterminazione finito, ne viene

$$
\lim _{x \rightarrow \infty} \frac{f^{\prime \prime}\left(x+b_{x}\right)}{f^{\prime \prime}(x)}=\mathrm{I}
$$

Ora si ha

$$
\alpha(x)=\frac{f(x+\mathrm{I})-f(x)}{f(x)}=\frac{f^{\prime}(x)}{f(x)}+\frac{f^{\prime \prime}(x)}{2 f(x)} \frac{f^{\prime \prime}(x+\theta)}{f^{\prime \prime}(x)}, \quad 0 \leq \theta \leq \mathrm{I} .
$$

In forza delle $(6 \mathrm{I})$ scriveremo quindi :

$$
\alpha(x)=\frac{f^{\prime}(x)}{f(x)}+\frac{f^{\prime \prime}(x)}{2 f(x)} \eta(x), \quad \lim _{x \rightarrow \infty} \eta(x)=\mathrm{r} .
$$

Poniamo

ne verrà

$$
\varphi(x)=\int_{x_{0}}^{x} \frac{f^{\prime \prime}(x)}{2 f(x)} \eta(x) d x
$$

$$
\int_{x_{0}}^{x} \alpha(x) d x=\lg f(x)-\lg f\left(x_{0}\right)+\varphi(x)
$$

cioè :

(64)

$$
\frac{f(x)}{\int_{x^{x_{0}}}^{x} \alpha(x) d x}=f\left(x_{0}\right) e^{-\varphi(x)} .
$$

Considerando che, per la terza delle (6o), si ha

$$
\left\{\begin{array}{l}
\varphi(x)=\sigma_{1}(\xi) \int_{x_{0}}^{x} \frac{f^{\prime}}{f} d x, \quad e^{\varphi(x)}=\left[\frac{f(x)}{f\left(x_{0}\right)}\right]^{\sigma_{1}(\xi)}, \\
x_{0} \leqslant \xi \leqslant x_{1}, \quad \sigma_{1}=\frac{n \sigma}{2}, \quad \lim _{\xi=\infty} \sigma_{1}(\xi)=0,
\end{array}\right.
$$

potremo scrivere: 


$$
\frac{[f(x)]^{1+\sigma_{x}(\xi)}}{\int_{x_{0}}^{x} \alpha(x) d x}=\left[f\left(x_{0}\right)\right]^{i+\sigma_{2}(\xi)} .
$$

Poichè la variabile $\sigma_{\mathrm{I}}(\xi)$, per ogni coppia $x_{0}, x$ è finita determinata e tende allo zero, per $x_{\mathrm{o}}=\infty$, si vede che le variabili $f(x), \int_{x_{0}}^{x} \alpha(x) d x$, per $x=\infty$ convergono o divergono insieme.

Fatto $x=x_{0}+n$, e ricordando la (57) concluderemo che: le variabili

$$
f(n)=\prod_{i=0}^{n}\left[\mathrm{I}+\alpha\left(x_{0}+r\right)\right], \int_{x_{0}}^{x_{0}+n} \alpha(x) d x,
$$

convergono o divergono insieme, cioè che: condizione necessaria e sufficiente perche sia convergente il prodotto infunito $\prod_{\mathrm{r}}^{\infty}[1+\alpha(x)]$, è che la funzione $\alpha(x)$ sia atta alla integrazione definita impropria nel tratto $\left(x_{0}, \cdots+\infty\right)$. Nel caso della divergenza l'ordine di infinito della variabile $f(x)$ si ottiene, con una prima approssimazione, calcolando quello della variabile $e^{\int_{x_{0}}^{x} a(x) d x}$, per $x=+\infty$.

32. Poniamo :

$$
\frac{f^{\prime}(x)}{f(x)}=\alpha(x) \delta(x), \quad f^{\prime}=f . \alpha \delta .
$$

Dalla formula (58), per le ipotesi ammesse al $\mathrm{n}^{\circ}$ precedente, dedurremo: $\lim _{x=\infty} \delta(x)=$ I. Derivando, e dividendo poi per $f$, avremo:

$$
\frac{f^{\prime \prime}}{f}=(\alpha \delta)^{2}+\frac{d}{d x}(\alpha \delta) \text {. }
$$

Poichè la $\alpha^{2}$ ha, per $x=+\infty$, il medesimo comportamento assintotico della $(\alpha \delta)^{2}$, si scorge che : Se la funzione $\alpha^{2}(x)$ è atta alla integrazione definita impropria nel tratto $(\mathrm{x}, \cdots+\infty)$, la funzione $\varphi(x)$ definita dalla formula (63) è finita per $x=+\infty$, e le due espressioni

$$
e^{\int_{x_{0}}^{x_{0}+n} \alpha(x) d x}, \quad f\left(x_{0}+n\right)=\prod_{r=0}^{n}\left[1+\alpha\left(x_{0}+r\right)\right]
$$

banno lo stesso ordine di infinito ${ }^{*}$ ). divergenti.

*) Cfr. il $\mathrm{n}^{\circ}$ xo, $\$$ III; si veda anche il $\mathrm{n}^{\circ} \mathrm{I}_{4}$ della Nota Sui prodotti infiniti 
33. Anche la $\alpha(x)$ sia atta alla integrazione impropria nel tratto $(1, \cdots+\infty)$. Ricordando la (57) potremo scrivere la (64) sotto la forma :

$$
\prod_{r=0}^{\infty}\left[1+\alpha\left(x_{0}+r\right)\right]=\left[1+\alpha\left(x_{0}\right)\right] \cdot e^{-\varphi(+\infty)} \cdot e^{\int_{x_{0}}^{+\infty} \alpha(x) d x} .
$$

Supponendo $\alpha\left(x_{0}\right)=0$, avremo:

$$
\prod_{r=0}^{\infty}\left[\mathrm{I}+\alpha\left(x_{0}+r\right)\right]=e^{-\varphi(+\infty)} e^{\int_{x_{0}}^{\infty} \alpha(x) d x} .
$$

Si consideri che

e, posto

$$
\varphi(+\infty)=\lim _{x=\infty} \int_{x_{0}}^{x} \frac{f^{\prime \prime}(x)}{2 f(x)} \eta(x) d x,
$$

$$
\int_{x_{0}}^{x} \frac{f^{\prime \prime}(x)}{2 f(x)} n(x) d x=\psi(x)-\psi\left(x_{0}\right)
$$

si osservi che, nella ipotesi in cui ci siamo posti, la $\psi(x)$ è, per $x=+\infty$, infinitesima; onde risulta

ed infine :

$$
\varphi(+\infty)=-\psi\left(x_{0}\right)
$$

$$
\prod_{r=0}^{\infty}\left[I+\alpha\left(x_{0}+r\right)\right]=e^{\psi\left(x_{0}\right)} \cdot e^{\int_{x_{0}}^{\infty} \alpha(x) d x} .
$$

Nella Nota Sui prodotti infiniti divergenti si è trascurato il fattore $e^{\psi\left(x_{0}\right)}$, il quale sarà tanto più prossimo ad I quanto più $x_{0}$ è grande: e si è scritta l'eguaglıanza approssimata :

$$
\prod_{r=0}^{\infty}\left[\mathrm{I}+\alpha\left(x_{0}+r\right)\right]=e^{\int_{x_{0}}^{+\infty} \alpha(x) d x} .
$$

Modena, 8 febbraio 1904.

ETTORE BORTOLOTTI. 